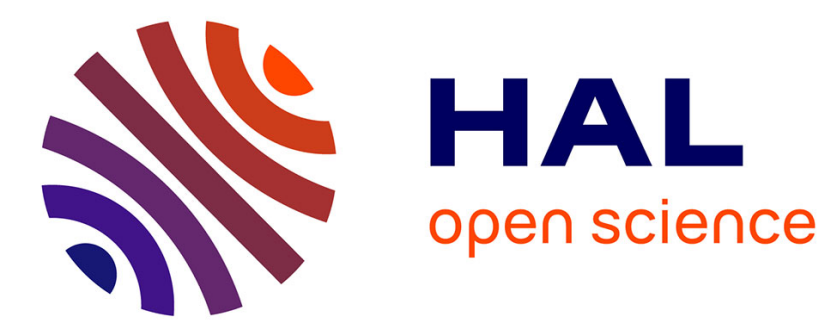

\title{
Blind precoding in line-of-sight MIMO channels
}

\author{
Paul Ferrand, Sheng Yang
}

\section{To cite this version:}

Paul Ferrand, Sheng Yang. Blind precoding in line-of-sight MIMO channels. 17th IEEE International Workshop on Signal Processing Advances in Wireless Communications (SPAWC 2016), Jul 2016, Edinburgh, United Kingdom. 10.1109/SPAWC.2016.7536764 . hal-01433727

\section{HAL Id: hal-01433727 \\ https://hal-centralesupelec.archives-ouvertes.fr/hal-01433727}

Submitted on 10 Jun 2020

HAL is a multi-disciplinary open access archive for the deposit and dissemination of scientific research documents, whether they are published or not. The documents may come from teaching and research institutions in France or abroad, or from public or private research centers.
L'archive ouverte pluridisciplinaire HAL, est destinée au dépôt et à la diffusion de documents scientifiques de niveau recherche, publiés ou non, émanant des établissements d'enseignement et de recherche français ou étrangers, des laboratoires publics ou privés. 


\title{
Blind Precoding in Line-of-Sight MIMO Channels
}

\author{
Paul Ferrand and Sheng Yang
}

\begin{abstract}
As we move towards densified networks for mobile access, with a larger number of access points, the need for high performance wireless backhaul increases drastically. In this respect, line-of-sight (LoS) multiple-input, multiple-output (MIMO) communication is a promising technique which can enable spatial diversity gains in such links, without increasing the allocated bandwith or overstepping requirements in radiated power. In this paper, we show that under practical conditions, the LoS MIMO channel matrix with dual-polarized (DP) antennas is block-circulant with circulant blocks (BCCB). This fact allows one to blindly diagonalize the channel, even in the absence of channel side-information (CSI) at the transmitter. In this case we show that appropriate precoding steps can greatly improve the performance of non-linear decoding in the LoS MIMO channel.
\end{abstract}

\section{INTRODUCTION}

In the evolution of wireless mobile communications towards the $5^{\text {th }}$ generation, the densification of the network is a key architecture proposal that requires small base stations or relays to be positioned all over the coverage area in order to get the network access points closer to the user. In many cases, such equipment cannot be expected to be linked to the global backbone of the communication network by a fixed line, due to cost or practical constraints. In this situation, wireless backhauling is the preferred technology, and has seen an increase of interest in the recent years. Such wireless backhaul links have a high demand in terms of data rates and reliability, which are generally handled by high-performance equipment with very directional antennas.

A way to improve the performance of such links is to use multiple antennas at both the receiver and transmitter side. Since the system is mainly in line-of-sight (LoS), the MIMO channel experienced on the link is very sparse and does not provide much diversity in itself [1], which is a deterrent in using MIMO technologies. A way to improve the MIMO channel is to use the spherical wavefront experienced in practice by the signal [2] and separate the antennas in the array as to have a row-orthogonal MIMO channel [3]. In this case, the channel is optimally equalized by matched-filtering. Such approaches have been detailed in multiple works over the year [3]-[6], where the authors derived the optimal antenna separation needed with respect to system parameters for many different setups. The potential gains are high even in realistic channel conditions [7], [8] where a diffuse channel contribution complements the LoS, specular component.

In practical cases, as we will show in this paper, the LoS MIMO channel is very structured, and this structure can

P. Ferrand is with the Mathematical and Algorithmic Sciences Lab, France Research Center, Huawei Technologies Co. Ltd., 92100 BoulogneBillancourt, France (email: paul.ferrand@huawei.com). S. Yang is with LSS, CentraleSupelec, 3 rue Joliot-Curie, 91190 Gif sur Yvette, France (email: sheng.yang@centralesupelec.fr). be harnessed to provide blind, feedback-less transmission strategies:

- In section II, we describe the LoS MIMO channel and its dual-polarized (DP) extension. We analyze the conditions for circularity of the channel in section III in the uniform linear array (ULA) case. Such structures also arise for uniform circular arrays (UCAs) [9], although in this case the only way to ensure circularity in non-trivial case is to have arrays facing each other perfectly.

- We then propose a number of results in section III which extends and generalize the well-known fact that Fourier matrices diagonalize circulant matrices regardless of the actual value of the coefficient. This enables blind or semiblind precoding as a viable transmission strategy in LoS MIMO links employing ULAs and UCAs.

- If some feedback is allowed, the optimal transmission strategy is a power and rate allocation over the separate data streams. However, for the truly blind case, we show in section IV that the performance is limited by the weakest mode in the LoS MIMO channel even when optimal maximum likelihood (ML) decoding is used. We show that performance can be vastly improved in this situation through algebraic precoding.

- In section $\mathrm{V}$, we analyze the strategies described in the paper in some cases of interest, and proceed towards conclusion.

In the sequel, we use $\boldsymbol{A}^{H}$ to denote the conjugate transpose of the complex matrix $\boldsymbol{A}$ and $\otimes$ to denote the Kronecker product of two matrices.

\section{SYSTEM MODEL}

We consider a MIMO channel represented by a channel matrix $\boldsymbol{H} \in \mathbb{C}^{n \times n}$, with a radio transceiver transmitting a symbol vector $\boldsymbol{x} \in \mathbb{C}^{n \times 1}$ through $n$ antennas towards a receiver reading symbols from $n$ antennas. The symbols received, denoted as $\boldsymbol{y} \in \mathbb{C}^{n \times 1}$, are corrupted by additive white gaussian noise $(\mathrm{AWGN}) \boldsymbol{z} \sim \mathcal{C N}\left(0, \sigma_{z}^{2}\right)$, i.e. each component of $\mathrm{z}$ follows an independent circularly symmetric Gaussian distribution of of variance $\sigma_{z}^{2}$. We thus have

$$
\boldsymbol{y}=\boldsymbol{H} \boldsymbol{x}+\boldsymbol{z}
$$

\section{A. The LoS MIMO channel model}

In contrast with more classical MIMO channels, the LoS MIMO model does not rely on random scattering effects to obtain a full-rank MIMO channel matrix $\boldsymbol{H}$, but rather on the pure separation and phase differences of the direct LoS paths arriving onto the receive MIMO array. Bohagen et al. derived the optimal parameters for linear arrays in [3]. We use here a different model, shown on Fig.1, using antenna heights rather 


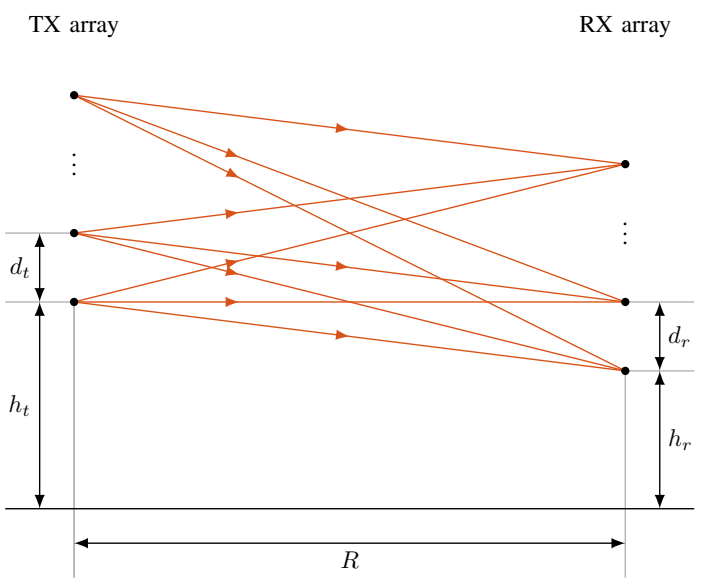

Fig. 1. Antenna array model and parameters for the ULA LoS MIMO channel.

than angles to parametrize the arrays beyond the spacing and inter-antenna distance. Note that the distance $r_{i, j}$ between the TX antenna $i \in\{1,2\}$ and the RX antenna $j \in\{1,2\}$ can be written as, with $g_{i, j}=\left(h_{t}+i d_{t}\right)-\left(h_{r}+j d_{r}\right)$

$$
r_{i, j}=\frac{R}{\cos \psi_{i, j}} \quad \psi_{i, j}=\arctan \left(\frac{g_{i-1, j-1}}{R}\right) .
$$

Globally, the pathloss will be the same for each antenna pairs, and the row-orthogonality of $\boldsymbol{H}$ is determined by the phase of its entries $h_{i, j} \propto \exp \left(\jmath 2 \pi r_{i, j} / \lambda\right)$, with $\lambda$ the carrier wavelength. Using successive small angle approximations, we can duplicate the derivation in [3] using our parametrization and derive the optimal inter-antenna distance for which $\boldsymbol{H}$ becomes row-orthogonal, which writes as

$$
d_{r} d_{t}=\frac{\lambda R}{n}=d_{\mathrm{opt}}^{2}
$$

for an $n$-antenna array and thus does not depend, based on first-order approximations, on the antenna heights.

\section{B. Polarization diversity}

In addition to the physical separation of antennas, one way to achieve orthogonality in the channel matrix is to use the polarization property of radio waves. From a theoretical point of view, a communication system could distinguish waves with respect to their polarization pattern. In practice, this can be achieved through linearly polarized antennas [10] which maximize their gain in the preferred polarization direction while minimizing their gain in the orthogonal direction. Polarization models are yet to be completely analyzed from a statistical point of view ; nonetheless, [11] presents a model gathering most of the results up to publication. We consider here their general model which we specialize for LoS MIMO channels, where in particular:

- Propagation relies more on line-of-sight rather than diffraction and reflections, which do present polarization selectivity. In this work, we assume that the so-called co-polar ratio (CPR) is set to $0 \mathrm{~dB}$, which means that vertically polarized waves and horizontally polarized waves experience the same pathloss.
- The cross-polar gain of the antennas used is very low and negligible, and depolarization effects from the channel are comparatively low.

- Anti-polar gains are arbitrary due to lack of further measurement and analysis on the topic.

Although the first and last hypothesis seem limiting at first, section III presents a proposition that effectively allows the analysis and results of this paper adapt to all DP channels. This leads in the end to consider a polarization matrix $\boldsymbol{X}$ as

$$
\boldsymbol{X}=\frac{1}{\sqrt{1+\chi}}\left(\begin{array}{cc}
1 & \sqrt{\chi} e^{\jmath \phi} \\
\mu \sqrt{\chi} e^{\jmath \phi} & 1
\end{array}\right)
$$

where $\phi$ is time-varying random phase, $\mu$ is a cross-polar arbitrary gain imbalance and $\chi$ is the global depolarization factor which represent the relative power of cross-polar components with respect to co-polar components. The normalization chosen here ensures that power is constant for non-zero values of $\chi$. Using this polarization matrix, we can update the $n \times n \operatorname{LoS}$ MIMO arrays considered earlier into $2 n \times 2 n$ arrays using the relation

$$
\boldsymbol{H}=\boldsymbol{X} \otimes \tilde{\boldsymbol{H}}
$$

\section{THE STRUCTURE OF THE LOS MIMO CHANNEL}

We start with some needed definitions about circulant and block-circulant matrices.

Definition 1. Let $\left\{a_{i}\right\}_{0 \leq i \leq n-1} \in \mathbb{C}^{n}$ be some sequence of complex numbers. A matrix $M \in \mathbb{C}^{n \times n}$ is called circulant if its elements $\left\{m_{i, j}\right\}_{0 \leq i, j \leq n-1}$ verify

$$
m_{i, j}=a_{k} \quad k=(n-1) \times i+j \bmod n .
$$

We can therefore write

$$
\boldsymbol{M}=\left(\begin{array}{cccc}
a_{0} & a_{n-1} & \cdots & a_{1} \\
a_{1} & a_{0} & \cdots & a_{2} \\
\vdots & & \ddots & \vdots \\
a_{n-1} & a_{n-2} & \cdots & a_{0}
\end{array}\right)
$$

and use $\boldsymbol{M}=\operatorname{circ}\left(a_{0}, \ldots, a_{n-1}\right)$ as a shorthand.

Definition 2. A matrix $M \in \mathbb{C}^{m n \times m n}$ is called block-circulant with circulant blocks (BCCB) if each row of blocks is a periodic shift of its previous row of blocks and every one on these blocks is a circulant matrix, e.g. $\boldsymbol{M}=\operatorname{circ}\left(\boldsymbol{M}_{1}, \ldots, \boldsymbol{M}_{m}\right)$ and each $\boldsymbol{M}_{i}$ is circulant for all $1 \leq i \leq m$.

\section{A. Circulant structure in LoS MIMO channels}

We consider the ULA model for an arbitrary number of antennas. The inter-antenna distances are equal and arbitrary, i.e. $d_{r}=d_{t}=d$, and we denote $h_{r}-h_{t}=\Delta h$. We can note at this point from Fig. 1 that this makes the arrays form a regular parallelogram in space. This leads to the following result:

Proposition 1. When $d_{r}=d_{t}=d$, the ULA LoS MIMO channel matrix has a Toeplitz structure.

The proof can be seen geometrically, as well as algebraically by noting that in this case, $g_{i, j}=\Delta h+(j-i) d$. We parametrize for the subdiagonals: let $0 \leq q<n$, then for all $0 \leq p<n-\delta$, 
the coefficients $g_{p, p+q}$ are equal. Symmetrically, so are the $g_{p+q, p}$. We further this result towards circulant channel matrices. In that case, since the channel is Toeplitz, we require that for any $0<p<n$, the $p^{\text {th }}$ coefficient of the first column is equal to the $(n-p)^{\text {th }}$ coefficient in the first row modulo $2 \pi$, as exemplified in definition 1 .

Proposition 2. A ULA LoS MIMO channel matrix is circulant iif the following is verified for all $0<p<n$, there exists $k \in \mathbb{Z}$ such that

$$
(n-2 p) d^{2}+2 d \Delta h=2 k \frac{\lambda R}{n}
$$

Proof. We expand (2) with the small angle approximation $\cos (x) \approx 1-x^{2} / 2$, which leads us to equire that for all $0<p<n$, there exists $k \in \mathbb{Z}$ such that

$$
\frac{2 \pi}{\lambda} R\left(1+\frac{\left(g_{0, n-p}\right)^{2}}{2 R^{2}}\right)=\frac{2 \pi}{\lambda} R\left(1+\frac{\left(g_{p, 0}\right)^{2}}{2 R^{2}}\right)+2 k \pi
$$

is verified. Using the fact that $g_{p, 0}=\Delta h-p d$ and $g_{p, 0}=$ $\Delta h+(n-p) d$ and expanding the results yields (8) after some algebra.

For the general case, the analysis of (8) is complex. We can nonetheless illustrate cases of interest where this condition may be enforced:

- For $d=d_{\mathrm{opt}}, \Delta h=0$ and an even $n$, meaning that we have optimal arrays facing each other, the condition reduces to

$$
(n-2 p) d_{\mathrm{opt}}^{2}+2 k \frac{\lambda R}{n}=0 .
$$

Since we have $d_{\mathrm{opt}}^{2}=\lambda R / n$ by (3) and $n$ even, $k=$ $(n-2 p) / 2$ is always an integer and thus verifies the above equation for all $0<p<n$. For odd $n$, the circulant property requires $d=\sqrt{2 k^{\prime}} d_{\text {opt }}$ for some integer $k^{\prime} \geq$ 1 for a similar property, thus leading to the fact that antenna arrays with an odd number of antennas are better conditioned in our applications if their separation is an even multiple of the original optimal separation.

- For $2 \times 2$ arrays, we can actually consider arrays that may not be optimally spaced and for which $d_{r}=d_{t}=\eta d_{\mathrm{opt}}$. In this case, we have only the case for $p=1$ to consider, for which (8) simplifies to

$$
\Delta h=k \frac{d_{\mathrm{opt}}}{\eta}
$$

and varying antenna height can help in conditioning the channel matrix towards circularity.

\section{B. Diagonalizing the LoS MIMO channel}

The following result from the literature shows that BCCB matrices can be diagonalized without knowing the exact values of their coefficients.

Theorem 1. BCCB matrices with $m$ blocks $\left(\boldsymbol{M}_{1}, \ldots, \boldsymbol{M}_{m}\right)$ of size $n \times n$ are simultaneously diagonalized by the Kronecker product of Fourier matrices $\boldsymbol{F}_{m} \otimes \boldsymbol{F}_{n}$. For some diagonal matrix $\boldsymbol{\Sigma}$ we have

$$
\boldsymbol{M}=\left(\boldsymbol{F}_{m}^{-1} \otimes \boldsymbol{F}_{n}^{-1}\right) \boldsymbol{\Sigma}\left(\boldsymbol{F}_{m} \otimes \boldsymbol{F}_{n}\right)
$$

Proof. See [12], specifically theorems 3.2.2 and 5.8.1.

This has a number of implications in practice for LoS MIMO communications where the base, single-polarized (SP) LoS MIMO channel verifies (8):

- For such channels, diagonalizing matrices are known at both sides of the communication for arbitrary realization of the channels, and thus optimal precoding and equalizations require no feedback.

- If the particular realization of the channel is needed, estimation through pilots is simplified since we only require the coefficient corresponding to the leading antenna element rather than the full channel matrix.

We recall the discussion in section II-B and from (4) we identify that the composite channel $\boldsymbol{X} \otimes \boldsymbol{H}$ is indeed BCCB when $\mu=1$ and can be blindly diagonalized through Theorem 1. When $\mu$ is arbitrary, we can make use of the following lemma to obtain a blind diagonalization.

Lemma 1. For any matrix $M$ such that

$$
\boldsymbol{M}=\left(\begin{array}{cc}
a & b \\
r e^{\jmath \theta} b & a
\end{array}\right)
$$

for some $(a, b) \in \mathbb{C}, r \in \mathbb{R}_{+}^{*}$ and $\theta \in[0,2 \pi]$, we have the following decomposition

$$
M=D^{-1} M^{\prime} D
$$

where

$$
\boldsymbol{M}^{\prime} \triangleq\left(\begin{array}{cc}
a & \sqrt{r} e^{\jmath \theta / 2} b \\
\sqrt{r} e^{\jmath \theta / 2} b & a
\end{array}\right) \quad \boldsymbol{D} \triangleq\left(\begin{array}{cc}
1 & 0 \\
0 & \sqrt{r} e^{\jmath \theta / 2}
\end{array}\right)
$$

Equivalently, in block form, for any matrix $M$ such that

$$
\boldsymbol{M}=\left(\begin{array}{cc}
\boldsymbol{A} & \boldsymbol{B} \\
r e^{\jmath \theta} \boldsymbol{B} & \boldsymbol{A}
\end{array}\right)
$$

for some $n \times n$ matrices $\boldsymbol{A}$ and $\boldsymbol{B}$, we have that

$$
\boldsymbol{M}=\left(\boldsymbol{D}^{-1} \otimes \boldsymbol{I}\right)\left(\begin{array}{cc}
\boldsymbol{A} & \sqrt{r} e^{\jmath \theta / 2} \boldsymbol{B} \\
\sqrt{r} e^{\jmath \theta / 2} \boldsymbol{B} & \boldsymbol{A}
\end{array}\right)(\boldsymbol{D} \otimes \boldsymbol{I}) .
$$

The proof follows by verification. Applying this lemma to the DP LoS MIMO channel matrix when $\mu=-1$ [11] allows to write

$$
\boldsymbol{H}=\left(\boldsymbol{D}^{-1} \otimes \boldsymbol{I}\right) \boldsymbol{H}^{\prime}(\boldsymbol{D} \otimes \boldsymbol{I})
$$

for

$$
\boldsymbol{D} \triangleq\left(\begin{array}{ll}
1 & 0 \\
0 & \jmath
\end{array}\right)
$$

Since $\boldsymbol{H}^{\prime}$ is circulant, it is diagonalizable and we have

$$
\boldsymbol{H}=\left(\left(\boldsymbol{F}_{2} \boldsymbol{D}\right)^{-1} \otimes \boldsymbol{F}_{n}^{-1}\right) \boldsymbol{\Sigma}\left(\boldsymbol{F}_{2} \boldsymbol{D} \otimes \boldsymbol{F}_{n}\right)
$$

for some diagonal matrix $\boldsymbol{\Sigma}$. As a side note, we can also use Lemma 1 to obtain a diagonalization for non-circulant $2 \times 2$ SP LoS MIMO channels and $4 \times 4$ DP LoS MIMO channels which can always be written as (12). 


\section{PRECODING FOR THE LOS MIMO CHANNEL}

From these results, we see that in a wide range of contexts, the LoS MIMO channel can be decomposed as $\boldsymbol{H}=\boldsymbol{U}^{H} \boldsymbol{\Sigma} \boldsymbol{U}$ for some diagonal matrix $\boldsymbol{\Sigma}$, possibly unknown at the transmitter, and orthogonal matrices $\boldsymbol{U}$, where $\boldsymbol{U}$ does not depend on the actual channel but only on a subset of environmental and system parameters. Due to the particular structure of the $\boldsymbol{U}$, the performance of un-precoded QAM may be limited by the minimum singular value of the channel matrix, as we will show next. This is true even with optimal ML detection. This is to be contrasted with the case of i.i.d. fading channel where the ML detection can recover all the receive diversity even with un-precoded QAM constellations.

\section{A. Performance analysis of un-precoded QAM}

The performance of the optimal ML detector depends on the minimum distance between constellation points at the receiver side. A commonly used upper bound of the error probability of detection is the sphere bound [13]

$$
P_{e} \leq \operatorname{Prob}\left\{\|\boldsymbol{z}\|>\frac{d_{\min }}{2}\right\}
$$

where $z$ is the AWGN and $d_{\min }$ is the minimum distance in the signal constellation at the receiver side. Since $\boldsymbol{H}=\boldsymbol{U}^{H} \boldsymbol{\Sigma} \boldsymbol{U}$ we have that

$$
\begin{aligned}
d_{\text {min }} & =\min _{\boldsymbol{x}_{A}, \boldsymbol{x}_{B} \in \mathrm{QAM}^{4}}\left\|\boldsymbol{\Sigma} \boldsymbol{U}\left(\boldsymbol{x}_{A}-\boldsymbol{x}_{B}\right)\right\| \\
& =\sigma_{\min } \min _{\substack{\boldsymbol{x}_{A}, \boldsymbol{x}_{B} \in \mathrm{QAM}^{4} \\
\boldsymbol{x}_{A}-\boldsymbol{x}_{B}=\alpha \boldsymbol{u}_{\min }}} \frac{1}{2} \sum_{i=1}^{4}\left|x_{A}(i)-x_{B}(i)\right| \\
& =2 \sigma_{\min } d_{\min , x}
\end{aligned}
$$

where the equality in (21) is verified if there exists $\boldsymbol{x}_{A}$ and $\boldsymbol{x}_{B}$ in the QAM constellation such that the difference is aligned with the row of $\boldsymbol{U}$ corresponding to the minimum singular value (and thus orthogonal with the others). In this equation $d_{\min , x}$ is the minimum distance in the QAM constellation, i.e.,

$$
\begin{aligned}
\frac{2(|\mathrm{QAM}|-1)}{3} & =\mathbb{E}\left|\frac{2 x}{d_{\min , x}}\right|^{2}=\frac{P}{n_{t}} \frac{4}{d_{\min , x}^{2}} \\
d_{\min , x} & =\sqrt{\frac{3 P}{2(|\mathrm{QAM}|-1)}} .
\end{aligned}
$$

Therefore, the probability of vector detection error is

$$
P_{e} \leq \operatorname{Prob}\left\{\|\boldsymbol{z}\|>\sigma_{\min } d_{\min , x}\right\}
$$

The condition on $\boldsymbol{x}_{A}$ and $\boldsymbol{x}_{B}$ is verified depending on environmental parameters and the particular form of $\boldsymbol{U}$. For denser constellations, (21) is verified with increasing probability regardless of the value of $\boldsymbol{U}$.

If a minimum mean-square error (MMSE) detector [1] is used, then the performance depends on the diagonal entries of the covariance of the MSE vector $\boldsymbol{Q}_{\mathrm{mmse}}$

$$
\boldsymbol{Q}_{\mathrm{mmse}}=\left(\frac{n_{t}}{P} \mathbf{I}+\boldsymbol{H}^{\mathrm{H}} \boldsymbol{H}\right)^{-1}=\boldsymbol{U}\left(\frac{n_{t}}{P} \mathbf{I}+\boldsymbol{\Sigma}^{\mathrm{H}} \boldsymbol{\Sigma}\right)^{-1} \boldsymbol{U}^{H}
$$

and therefore

$$
\operatorname{diag}\left(\boldsymbol{Q}_{\text {mmse }}\right)=\sum_{i=1}^{4}\left(\frac{n_{t}}{P}+\sigma_{i}^{2}\right)^{-1} \mathbf{I} .
$$

From the above expression, we can see that each stream have the same MSE and therefore the same performance. Further more, the MSE is a sum that is dominated by the term related to the smallest singular value $\sigma_{\min }$ of the channel matrix, i.e.,

$$
\left(\frac{n_{t}}{P}+\sigma_{\min }^{2}\right)^{-1} \leq \sum_{i=1}^{4}\left(\frac{n_{t}}{P}+\sigma_{i}^{2}\right)^{-1} \leq 4\left(\frac{n_{t}}{P}+\sigma_{\min }^{2}\right)^{-1}
$$

Therefore, the probability of symbol detection error is

$$
\begin{aligned}
P_{e} & \leq \operatorname{Prob}\left\{\left|\tilde{z}_{i}\right|>\frac{d_{\min , x}}{2 \alpha \sqrt{\left(\frac{n_{t}}{P}+\sigma_{\min }^{2}\right)^{-1}}}\right\} \\
& =\operatorname{Prob}\left\{\left|\tilde{z}_{i}\right|>\beta \sigma_{\min } d_{\min , x}\right\}
\end{aligned}
$$

where $\beta=\frac{1}{2 \alpha} \sqrt{1+\frac{n_{t}}{P \sigma_{\min }^{2}}}$.

\section{B. Blind precoding}

We recall that the similarity of the performance originated from the particular structure of the right singular matrix $\boldsymbol{U}$ for which the minimum distance of an un-precoded QAM depends only on the smallest singular value. Now, it becomes clear that one may be able to improve the performance by increasing the minimum distance through a precoding procedure. That is, we change the right singular matrix with precoding. Without loss of generality, we consider the following signal model:

$$
\boldsymbol{U} \boldsymbol{y}=\boldsymbol{\Sigma}(\boldsymbol{U} \boldsymbol{x})+\boldsymbol{U} \boldsymbol{z}=\boldsymbol{\Sigma} \tilde{\boldsymbol{x}}+\tilde{\boldsymbol{z}}
$$

where $\tilde{\boldsymbol{x}} \triangleq \boldsymbol{U} \boldsymbol{x}$ and $\tilde{\boldsymbol{z}}$ is i.i.d. AWGN. Since $\boldsymbol{U}$ is constant and known to the transmitter, one can design $\tilde{\boldsymbol{x}}$ from scratch. Note that the above channel model is equivalent to a parallel channel that is well studied in the literature.

- If $\Sigma$ is known to the transmitter, it is optimal to send independent streams with different entries of $\tilde{\boldsymbol{x}}$, under waterfilling power allocation. It means that the realization of $\boldsymbol{\Sigma}$ should be fed back to the transmitter.

- If $\Sigma$ is not known to the transmitter, then the productdistance criterion is known to be an efficient precoding construction metric [14].

We focus on the non-trivial case where $\Sigma$ is not known to the transmitter. With the product-distance criterion, a welladopted way to construct good unitary precoder is the algebraic construction from number theory. To date, the best known $4 \times 4$ algebraic rotation that gives the largest minimum productdistance is the following [15]

$$
\boldsymbol{T}=\left[\begin{array}{rrrr}
-0.3664 & -0.7677 & 0.4231 & 0.3121 \\
-0.2264 & -0.4745 & -0.6846 & -0.5050 \\
-0.4745 & 0.2264 & -0.5050 & 0.6846 \\
-0.7677 & 0.3664 & 0.3121 & -0.4231
\end{array}\right] .
$$

Thus, we shall use this rotation in such a way that $\tilde{\boldsymbol{x}}=\boldsymbol{T} \boldsymbol{s}$ where $s$ is a vector of uncoded QAM symbols, i.e.,

$$
\boldsymbol{x}=\boldsymbol{U}^{\top} \boldsymbol{T} \boldsymbol{s}=\boldsymbol{V} \boldsymbol{s}
$$

with $\boldsymbol{V} \triangleq \boldsymbol{U}^{\top} \boldsymbol{T}$ being the proposed global precoder. 


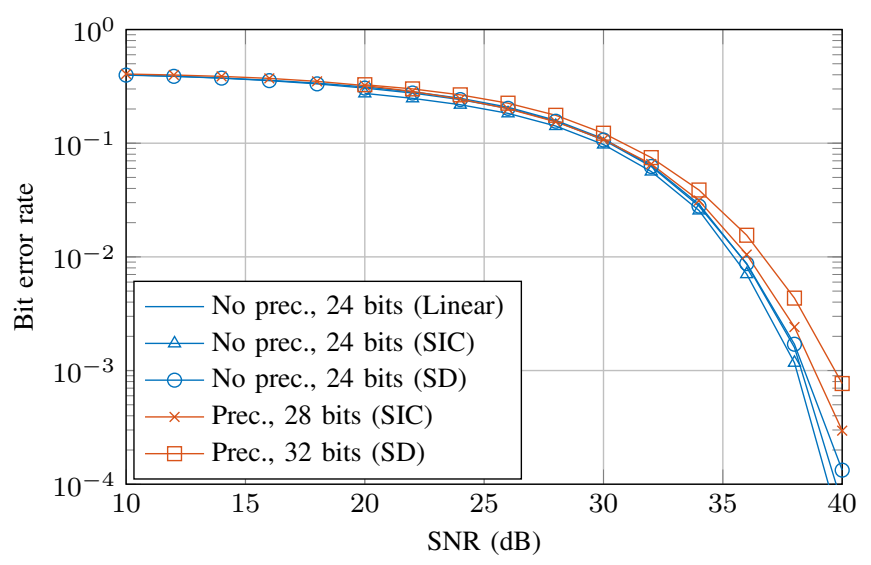

Fig. 2. Comparison of the average stream bit error rate (BER) for an known DP LoS MIMO ULA channel at the transmitter, with inter-antenna distance $d=0.33 d_{\mathrm{opt}}$ and different decoding and precoding architectures. The rate of each stream is adapted when precoding is used, as to achieve a BER of $10^{-3}$

\section{NUMERICAL RESULTS}

We analyze the performance of the precoding approaches in the two situations where the transmitter may adapt its transmission rate by any means, as well as when the transmitter has absolutely no information, with thus completely blind precoding. For both situations, we analyze linear and nonlinear decoding approaches, using either successive interference cancellation (SIC) [1] or an adapted sphere-decoding (SD) algorithm [16]. The latter is known to approach the optimal ML performance in practical setups.

When the transmitter is able to adapt the rate of each stream through limited feedback (Fig.2), for example using predefined adaptive modulation and coding schemes with increasing rate over time, the precoding allows to theoretically pack more bits in each MIMO symbol transmitted. We can see that without precoding, and as predicted in section IV, the performance of non-linear SIC and SD detection is similar to the performance of basic linear equalization. Using precoding allows to separate the data streams and improve the performance, packing up to

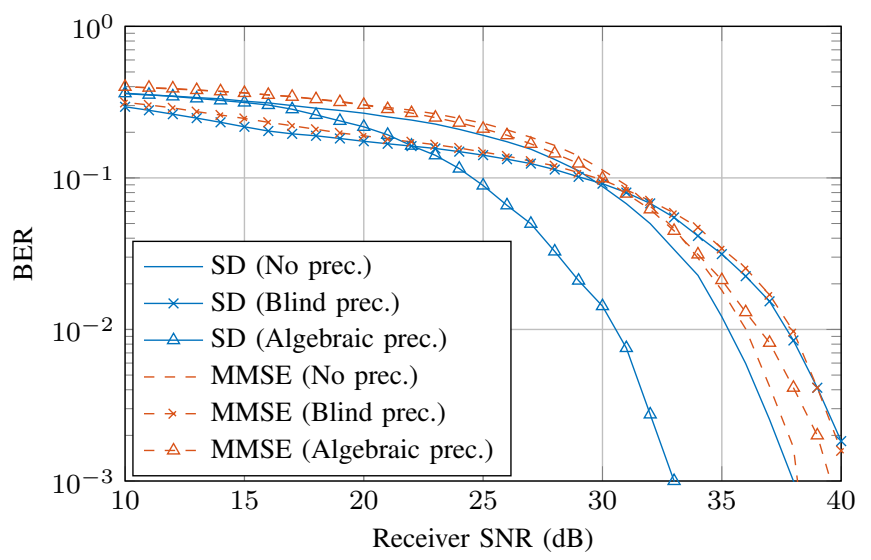

Fig. 3. Comparison of the average stream bit error rate (BER) for an unknown DP LoS MIMO ULA channel at the transmitter, with inter-antenna distance $d=0.33 d_{\text {opt }}$ and different decoding and precoding architectures. Each stream supports a 64-QAM, with thus 6 bits per stream.
$33 \%$ more bits per symbol. When the channel is unknown at the transmitter however (Fig.3), precoding in itself is actually detrimental to the performance of the system, with a loss up to $3 \mathrm{~dB}$ in SNR for the non-linear SD detection. In this situation, we have to employ an additional algebraic precoding step, as described in section IV, which enables a $5 \mathrm{~dB}$ gain for the non-linear detection approach. Algebraic precoding only restores the no-precoding performance for the linear detection approach.

\section{CONCLUSION}

In this paper, we studied the LoS MIMO channel, and detailed the necessary environmental parameters for the channel to become circulant. We proceeded to show that when using DP antennas in either linear or circular arrays, the channel could be blindly diagonalized using Kronecker products of Fourier matrices. We then showed that this particular structure in the channel makes non-linear detection perform badly. We finally described precoding steps that allows to improve the performance of the system greatly in cases where the transmitter is completely blind to the channel. Outlooks for this work lie in evaluating the robustness of the blind precoding approaches in practical environments, and whether one could design openloop calibration of the antenna arrays to compensate for operational mishaps like misalignment or wind turbulences.

\section{REFERENCES}

[1] D. N. C. Tse and P. Viswanath. Fundamentals of Wireless Communications. Cambridge University Press, 2005.

[2] J.-S. Jiang and M.A. Ingram. Spherical-wave model for short-range MIMO. IEEE Trans. Commun., 53(9):1534-1541, September 2005.

[3] F. Bohagen, P. Orten, and G.E. Oien. Construction and capacity analysis of high-rank line-of-sight MIMO channels. In IEEE Wireless Commun. and Networking Conf. (WCNC). IEEE, 2005.

[4] I. Sarris and A.R. Nix. Design and performance assessment of highcapacity MIMO architectures in the presence of a line-of-sight component. IEEE Trans. Veh. Technol., 56(4):2194-2202, 2007.

[5] L. Liu, W. Hong, H. Wang, G. Yang, N. Zhang, H. Zhao, J. Chang, C. Yu, X. Yu, X. Tang, H. Zhu, and L. Tian. Characterization of line-ofsight MIMO channel for fixed wireless communications. IEEE Antennas Wireless Propag. Lett., 6:36-39, 2007.

[6] X. Song and G. Fettweis. On spatial multiplexing of strong line-of-sight MIMO with 3D antenna arrangements. IEEE Wireless Commun. Letters, 4(4):393-396, 2015.

[7] M. Matthaiou, P. de Kerret, G. K. Karagiannidis, and J. A. Nossek. Mutual information statistics and beamforming performance analysis of optimized LoS MIMO systems. IEEE Trans. Commun., 58(11):3316âĂŞ3329, Nov 2010.

[8] P. Wang, Y. Li, X. Yuan, L. Song, and B. Vucetic. Tens of gigabits wireless communications over E-Band LoS MIMO channels with uniform linear antenna arrays. IEEE Trans. Wireless Commun., 13(7):3791-3805, 2014.

[9] Liang Zhou and Y. Ohashi. Low complexity millimeter-wave los-MIMO precoding systems for uniform circular arrays. In IEEE Wireless Commun. and Networking Conf. (WCNC), pages 1293-1297, 2014.

[10] C. A. Balanis. Antenna Theory: Analysis and Design. Wiley, 2005.

[11] B. Clerckx and C. Oestges. MIMO Wireless Networks. Wiley, 2012.

[12] P. J. Davis. Circulant Matrices. American Mathematical Society, 1994.

[13] B. Hassibi and H. Vikalo. On the sphere-decoding algorithm i. expected complexity. IEEE Trans. Signal Process., 53(8):2806-2818, 2005.

[14] J. Boutros and E. Viterbo. Signal space diversity: a power-and bandwidthefficient diversity technique for the rayleigh fading channel. IEEE Trans. Inf. Theory, 44(4):1453-1467, 1998.

[15] E. Viterbo. http://www.ecse.monash.edu.au/staff/eviterbo/rotations/rotations.html. Retrieved Feb. 2016.

[16] E. Viterbo and J. Boutros. A universal lattice code decoder for fading channels. IEEE Trans. Inf. Theory, 45(5):1639-1642, 1999. 